\title{
Meningitis as a Hidden Cause of Neurological Deterioration in Patients With Known Brain Metastases: A Report of Two Cases
}

\author{
Ioannis Vrettos ${ }^{\mathrm{a}}$, Panagiota Voukelatou ${ }^{\mathrm{a}, \mathrm{b}}$, Stavros Fokas ${ }^{\mathrm{a}}$, Athina Bitsikokou ${ }^{\mathrm{a}}$, \\ Athanasios Didaskalou ${ }^{a}$, Andreas Kalliakmanis ${ }^{\mathrm{a}}$
}

\begin{abstract}
When a patient with known brain metastases presents to the emergency department with neurological deterioration, an overt diagnosis exists. However, alternative diagnoses should also be considered in the appropriate clinical context. A correct diagnosis is critical for the appropriate treatment, especially in cases, in which a reversible cause of illness exists. In this report, we describe two cases of cancer patients with known brain metastases in whom the neurological deterioration was due to carcinomatous meningitis and viral menigitis, respectively. A lumbar puncture was performed based on the fact that neurological deterioration was in contrast with the absence of new findings in brain CT scans. As it is emphasized by these two cases, patients with brain metastases, unchanged at $\mathrm{CT}$ imaging, and recent neurological deterioration must undergo lumbar puncture before their symptoms are considered a progression of their already existing brain metastases. In some cases, the deterioration might be due to a potentially reversible illness or due to an illness requiring specific treatment.
\end{abstract}

Keywords: Cancer; Brain metastases; Fever; Altered mental status; Headache; Meningitis

\section{Introduction}

Brain metastases occur in about $25 \%$ of all cancer patients during the course of their illness. In adults, most brain metastases originate from lung cancers $(50-60 \%)$, followed by breast cancers $(15-20 \%)$, melanomas $(5-10 \%)$ as much as cancers of the gastrointestinal tract and renal cell carcinomas [1].

Neurological manifestations occur in more than two-thirds

Manuscript accepted for publication August 30, 2016

a2nd Department of Internal Medicine, General and Oncology Hospital of Kifissia "Agioi Anargyroi", Greece

${ }^{\mathrm{b}}$ Corresponding Author: Panagiota Voukelatou, 2nd Department of Internal Medicine, General and Oncology Hospital of Kifissia “Agioi Anargyroi”, Timiou Stavrou 14, Athens 14564, Greece.

Email: tina.voukelatou@gmail.com

doi: http://dx.doi.org/10.14740/jmc2631w of patients with brain metastases. The clinical presentations include headache and vomiting (with or without nausea), altered mental status, ataxia, seizures, dizziness, hemiparesis, aphasia, visual reduction, cranial nerve impairment, etc. In some cases, the onset of neurological manifestations is abrupt like a stroke attack [2]. Moreover, fever may coexist both as a manifestation of cancer and also from the brain metastases itself [3].

When a patient with known brain metastases presents to the emergency department (ED) with one, or some, of these symptoms, an overt diagnosis exists. However, alternative diagnoses should also be considered in the appropriate clinical context. For example, symptoms like headache, nausea and vomiting, seizures, motor or sensory focal deficit and altered mental status may also occur in patients with meningitis [4]. Consequently, a correct diagnosis is critical for the appropriate treatment, especially in cases, in which a reversible cause of illness exists.

In this report, we describe two cases of cancer patients with known brain metastases in whom the neurological deterioration was due to meningitis.

\section{Case Reports}

\section{Case 1}

A 53-year-old female patient was brought to the ED due to excessive vomiting, headache, confusion and generalized malaise during the last few days. From her past medical history, she was diagnosed with invasive ductal carcinoma (IDC) stage 4 with bone metastases for which she received chemotherapy. A few days later, she underwent a brain magnetic resonance imaging (MRI) and she was diagnosed to have a mild ventricular distention and multiple small sized parenchymal metastases. From her neurological examination on admission, nuchal stiffness and altered mental status were observed while her inflammatory markers were the following: C-reactive protein $(\mathrm{CRP})<3.27 \mathrm{mg} / \mathrm{L}(0-5 \mathrm{mg} / \mathrm{L})$, erythrocyte sedimentation rate (ESR) $2 \mathrm{~mm} / \mathrm{h}(2-20 \mathrm{~mm} / \mathrm{h})$ and white blood cells (WBCs) $21.760 \times 10^{3} / \mu \mathrm{L}\left(4.000-11.000 \times 10^{3} / \mu \mathrm{L}\right)$, and polymorphonuclear neutrophils (PMNs) $18.460 \times 10^{3} / \mu \mathrm{L}(2.500$ $\left.-7.500 \times 10^{3} / \mu \mathrm{L}\right)$. She underwent a brain CT with intravenous contrast medium that revealed a mild ventricular distention without any widening of the peripheral subarachnoid spaces. 
As the brain computed tomography (CT) could not explain completely the clinical picture, she underwent lumbar puncture, which revealed a clear and colorless cerebrospinal fluid (CSF) with the presence of six cells, glucose of $25 \mathrm{mg} / \mathrm{dL}$ (serum glucose of $97 \mathrm{mg} / \mathrm{dL}$ ), lactate dehydrogenase (LDH) of $504 \mathrm{U} / \mathrm{L}$ and protein of $250 \mathrm{mg} / \mathrm{dL}$. From the CSF cytology, the presence of tumor cells was confirmed. She was treated with dexamethasone with gradual improvement of her clinical symptoms and finally she was referred to her oncologist for further treatment.

\section{Case 2}

A 56-year-old male patient was admitted due to generalized malaise, headache, altered mental status, vomiting, nausea and fever $\left(38.5^{\circ} \mathrm{C}\right)$ for the last few days. On admission, his laboratory findings were the following: WBCs $5.900 \times 10^{3} / \mu \mathrm{L}(4.000$ $\left.-11.000 \times 10^{3} / \mu \mathrm{L}\right)$, PMNs $5.500 \times 10^{3} / \mu \mathrm{L}(2.500-7.500 \times$ $\left.10^{3} / \mu \mathrm{L}\right), \mathrm{CRP} 7.68 \mathrm{mg} / \mathrm{L}(0-5 \mathrm{mg} / \mathrm{L})$ and ESR $102 \mathrm{~mm} / \mathrm{h}(2$ - $20 \mathrm{~mm} / \mathrm{h}$ ). From his past medical history, he was diagnosed with squamous cell lung carcinoma with brain metastases for which he received both chemotherapy and radiotherapy. The last days, before his current neurological deterioration and until the time of admission, he started dexamethasone in order to treat the brain peritumoral edema. Based on his medical history, he underwent a brain CT scan, which revealed brain metastases (the bigger one was located near cerebellum) with accompanied vasogenic edema. He was treated with dexamethasone and mannitol. Because of persistent headache and fever, in the absence of an obvious site of infection, coexisting meningitis was suspected. He underwent a lumbar puncture with the following CSF characteristics: clear and colorless, negative Gram and Ziehl-Neelsen stains, CSF cell count: 80 cells (54\% PMNs and 46\% lymphocytes), LDH of $69 \mathrm{U} / \mathrm{L}$, glucose of $60 \mathrm{mg} / \mathrm{dL}$ (serum glucose of $139 \mathrm{mg} / \mathrm{dL}$ ), and protein of $180.81 \mathrm{mg} / \mathrm{dL}$. In the absence of titers for virus-specific antibodies in CSF, he was empirically treated with acyclovir. His symptoms improved rapidly and fever receded. After 5 days, he underwent a new lumbar puncture with the following results: CSF cell count of two cells, LDH of $46 \mathrm{U} / \mathrm{L}$, glucose of $130 \mathrm{mg} / \mathrm{dL}$ (serum glucose of $203 \mathrm{mg} / \mathrm{dL}$ ), and protein of $183.11 \mathrm{mg} / \mathrm{dL}$. Two weeks after his admission, while the patient was asymptomatic, he underwent a brain MRI scan that revealed the already known metastases with accompanied peritumoral edema.

\section{Discussion}

In the first case, the neurological deterioration was due to a non-reversible cause. The infiltration of the leptomeninges by malignant cells, as a complication of cancer, is called carcinomatous meningitis and breast cancer is the most common solid tumor complicated by meningeal carcinomatosis at a proportion of approximately 5\% [5]. Although the overall prognosis remains poor, the expansion of the repertoire of available drugs for intrathecal administration, systemic chemotherapy, gene therapy and the use of immunotoxins for the management of leptomeningeal metastases can prolong life expectancy and improve the health-related quality of life in these patients [6]. To these treatment options, the craniospinal radiotherapy should also be added, which is feasible and effective for palliative treatment of leptomeningeal metastases [7].

In the second case, the neurological deterioration was due to a self-limiting, potentially reversible [8] and, in cases of bacterial meningitis, treatable illness (especially if the appropriate treatment initiates in time) [9].

Our patients had both negative Kernig's and Brudzinski's sign, while only the first one had nuchal rigidity. Nevertheless, it has been reported that nuchal rigidity was found during clinical examination in $35 \%$ and $13 \%$ of the acute care geriatric and younger patients, respectively, despite the absence of meningitis [10]. Anyway, the three classic meningeal signs (Kernig's sign, Brudzinski's sign and nuchal rigidity) were not able to accurately discriminate patients with meningitis from those without [4] and individual items of the clinical history have low accuracy for the diagnosis of meningitis [11]. However, in patients with suspected meningitis, rapid and accurate clinical evaluation is required in order to determine the need for lumbar puncture [4].

The main reason that leads to the decision to perform a lumbar puncture in our patients was the fact that their neurological deterioration was in contrast with the absence of new findings in the performed brain CT scans. This fact, combined with the presence of headache and altered mental status, raised the suspicion of meningitis.

As it is emphasized by these two cases, patients with brain metastases, unchanged at CT imaging, and recent neurological deterioration must undergo lumbar puncture before their symptoms are considered a progression of their already existing brain metastases. In some cases, the deterioration might be due to a potentially reversible illness or due to an illness requiring specific treatment.

\section{Financial Support}

No financial support was given for this publication.

\section{Conflicts of Interest}

None of the authors has any conflicts of interest.

\section{References}

1. Johnson JD, Young B. Demographics of brain metastasis. Neurosurg Clin N Am. 1996;7(3):337-344.

2. Zhang X, Zhang W, Cao WD, Cheng G, Liu B, Cheng J. A review of current management of brain metastases. Ann Surg Oncol. 2012;19(3):1043-1050.

3. Johnson M. Neoplastic fever. Palliat Med. 1996;10(3):217224.

4. Thomas KE, Hasbun R, Jekel J, Quagliarello VJ. The di- 
agnostic accuracy of Kernig's sign, Brudzinski's sign, and nuchal rigidity in adults with suspected meningitis. Clin Infect Dis. 2002;35(1):46-52.

5. Pavlidis N. The diagnostic and therapeutic management of leptomeningeal carcinomatosis. Ann Oncol. 2004;15(Suppl 4):iv285-291.

6. Sagar SM. Carcinomatous Meningitis: it does not have to be a death sentence. Oncology (Williston Park). 2002 Feb;16(2):237-243; discussion 244, 249-250.

7. Hermann B, Hultenschmidt B, Sautter-Bihl ML. Radiotherapy of the neuroaxis for palliative treatment of leptomeningeal carcinomatosis. Strahlenther Onkol.
2001;177(4):195-199.

8. Logan SA, MacMahon E. Viral meningitis. BMJ. 2008;336(7634):36-40.

9. Moller K, Skinhoj P. Guidelines for managing acute bacterial meningitis in adults. West J Med. 2000;173(4):223224.

10. Puxty JA, Fox RA, Horan MA. The frequency of physical signs usually attributed to meningeal irritation in elderly patients. J Am Geriatr Soc. 1983;31(10):590-592.

11. Attia J, Hatala R, Cook DJ, Wong JG. The rational clinical examination. Does this adult patient have acute meningitis? JAMA. 1999;282(2):175-181. 